\title{
A xiloteca do Centro de Pesquisas do Cacau e as madeiras da Mata Atlântica
}

\author{
The cocoa research center's xylotheque and the woods of the Atlantic Forest \\ Mara Lúcia A. Valle $1^{\oplus \infty}$, Bianca de Sousa Aleluia Santos $2^{\circledR}$ \& Jomar G. Jardim $1^{\oplus}$
}

1. Centro de Formação em Ciências Agroflorestais, Universidade Federal do Sul da Bahia, Campus Jorge Amado, Itabuna, Bahia, Brasil e Herbário Centro de Pesquisas do Cacau - CEPEC/CEPLAC

\section{Graduanda de Engenharia Flores-} tal, Centro de Formação em Ciências Agroflorestais, Universidade Federal do Sul da Bahia, Campus Jorge Amado,Itabuna, Bahia, Brasil

\section{Palavras-chave}

Madeira. Espécies florestais. Coleções botânicas. Coleções de madeira.

\section{Keywords}

Wood. Forest species. Botanical collections. Wood Collections.

\section{Doi}

doi.org/10.33447/paubrasilia.v2i2.28

Recebido em: 19/08/2019

Aceite em: 18/10/2019

\section{Resumo}

Xiloteca é uma coleção científica de madeiras identificadas com dados de coleta disponíveis, e que é representativa da diversidade biológica. É um referencial para estudos e pesquisas na área botânica e tecnológica, tanto para produtores como para comerciantes de madeira, servindo também para comparação e identificação de novas amostras de madeira. A xiloteca em estudo está associada ao herbário do Centro de Pesquisas do Cacau, que atualmente é um dos maiores da região nordeste e conserva a mais importante coleção de espécimes vegetais representantes da Mata Atlântica do sul baiano e norte do Espírito Santo. O objetivo desse estudo é dar visibilidade às informações presentes nessa xiloteca. Com o aumento da visibilidade da xiloteca para uma maior audiência - pesquisadores e público em geral -, espera-se valorizar informações sobre o patrimônio das espécies vegetais do sul da Bahia, resguardando dados de importante relevância para a compreensão da diversidade florestal da região.

\begin{abstract}
A xylotheque is a scientific collection of identified woods with collection data available representative of the biological diversity. It is a reference tool for studies and research in the botanical and technological area, both for wood producers and traders, also aiming at comparison and identification of new samples of wood. The xylotheque described hereafter is associated with the herbarium of the Cocoa Research Center, which is currently one of the largest in the Northeast region and holds the most important collection of plant specimens of the Atlantic Forest of Southern Babia and Northern Espirito Santo. The aim of this study is to give visibility to the information available in the xylotheque. With the increase of the visibility of the xylotheque to a wider audience - researchers and public in general -, we expected to give value to information on the patrimony of the vegetal species of the south of Babia, safeguarding data of important relevance for the understanding of the forest diversity of the region.
\end{abstract}

\section{Introdução}

Xiloteca é uma coleção científica de madeira identificada e com dados de coleta disponíveis. Constitui um referencial para desenvolver pesquisas na área de taxonomia, anatomia, genética e tecnologia, servindo também como base de informações para produtores e comerciantes de madeira, e para a formação de recursos humanos.

Uma xiloteca tem como objetivo conservar amostras de madeiras de espécies locais, regionais ou de um continente. Pode auxiliar estudantes e pesquisadores em suas pesquisas e na solução de problemas, como em comercialização de madeiras, anatomia da madeira, monitoramento de planos de manejo e do controle do desmatamento ilegal, entre outros (Melo Junior et al., 2014; Barros; Coradin, 2016). Além disso, pode ser utilizada como fonte de informação para a botânica forense na solução de problemas criminais (Coyle et al., 2005), assim como subsidiar os órgãos fiscalizadores na correta identificação de amostras de madeira. 
Existem 37 xilotecas em funcionamento no Brasil, sendo que a região nordeste é a menos representada, com apenas duas, localizadas no estado da Bahia (Barros; Coradin, 2016): a xiloteca Professor José Pereira de Sousa, da Universidade Federal da Bahia, criada em 2012, e a xiloteca do Centro de Pesquisa do Cacau (CEPEC), que é mais antiga. Ambas estão indexadas no Index Xylariorum (Lynch; Gasson, 2016), demonstrando sua importância para o país e para o mundo.

Geralmente, as xilotecas estão associadas a herbários já estruturados, mantendo-se o mesmo acrônimo do herbário acrescido da letra "w", em referência a wood. Porém, nem sempre os espécimes de madeiras depositados na xiloteca ligada ao herbário estão relacionados aos espécimes vegetais depositadas no mesmo, uma vez que essa prática começou a ser desenvolvida apenas nas últimas duas décadas (Vásquez-Correa, 2017). Contudo, o ideal é que se tenha sistematicamente essa associação e é importante que se faça tal investigação entre herbário e xiloteca a ele associado.

Não é exagero afirmar que as coleções constantes nos herbários e xilotecas, bem como os profissionais associados a eles, tornaram-se recentemente de importância fundamental para cumprir parte dos desafios da humanidade para o novo milênio, como a conservação das espécies vegetais, principalmente as ameaçadas de extinção (Sobral; Stehmann, 2009; Forzza et al., 2012). O sul da Bahia, uma das regiões mais diversas do planeta (Mori et al., 1981; Thomas et al., 1998; Martini et al., 2007; Amorim et al., 2008), tem na xiloteca e no herbário um importante papel na conservação dos espécimes e, consequentemente, na preservação da história botânica da região e do estado. Destaca-se que, para a produção científica, a conservação de testemunhas de madeiras identificadas possibilita a troca de dados entre pesquisadores, facilitando o desenvolvimento de pesquisas em parceria. Além disso, permite que as informações oriundas das pesquisas possam ser disponibilizadas para o público em geral (Dagnino, 2008).

Assim, este estudo tem como objetivo apresentar o atual estado do conhecimento sobre a xiloteca do CEPECw, indicando as ações futuras para sua conservação, ampliação e divulgação.

\section{Histórico}

A xiloteca do CEPECw é associada ao herbário CEPEC, situado no Km 22 da rodovia BR 415, Ilhéus/Itabuna e recebeu, em homenagem, o nome alternativo de Herbário "André Maurício V. de Carvalho" (Amorim, 2015). Esta coleção foi criada em 1965 com o propósito de documentar a flora do sul da Bahia, região ainda pouco representativa em coleções de herbários nacionais e internacionais (Mori; Silva, 1979), naquela época. Contudo, com o aumento do intercâmbio e o interesse de diversos pesquisadores na flora da Bahia, sua área de abrangência foi ampliada, incluindo amostras de outros estados, sendo hoje uma das maiores referências para estudos com foco na Mata Atlântica. Atualmente, o herbário CEPEC é o segundo maior herbário do nordeste do Brasil, com aproximadamente 160 mil espécimes (exsicatas), sendo mais de 3.000 mil tipos-nomenclaturais - espécimes usados para propor novos taxa) -, muitos desses exclusivos do sul da Bahia e, por isso, de importância inestimável para a ciência e para a humanidade.
A coleção de madeiras foi iniciada por coletores da Comissão Executiva do Plano da Lavoura Cacaueira (CEPLAC), que na época realizavam expedições para coleta de espécimes para o herbário, coletando também amostras de madeira (Mori; Silva, 1979).

A maioria das amostras de madeira constantes no CEPECw foram coletadas na década de 1970, quando houve a expansão do cultivo de cacau (Theobroma cacao L.) na Bahia e no Espírito Santo, promovido pela CEPLAC. Na oportunidade da derrubada da floresta para formar os plantios, as equipes do herbário coletavam as amostras de madeira (Santos TS, com. pess.). Por isso, muitas das amostras não possuem uma exsicata associada no herbário. Assim, as amostras foram organizadas e listadas a partir do nome popular e, posteriormente, relacionadas aos nomes científicos e respectivas famílias, quando possível.

\section{Material e Métodos}

Foi realizado o levantamento de todas os espécimes presentes na coleção, com anotação dos nomes - seja vernacular ou científico - presentes em cada amostra. A partir daí, realizou-se uma busca no banco de dados do herbário para encontrar os materiais testemunhos e atualizar as identificações dos nomes científicos. Quando não existiam os materiais testemunhos, os nomes vernaculares foram utilizados para atribuir a identificação a partir da experiência dos autores ou usando como base Silva et al. (2008).

\section{Resultados e Discussão}

A xiloteca conta atualmente com 367 amostras, identificadas a partir do nome vernacular e até o nível de espécie, quando possível (Tabela 1). Embora seja uma xiloteca antiga, ainda é pequena, talvez, pela falta de profissionais atuando na área de madeira no auge da coleta de material biológico e, por isso, a xiloteca tenha se estabilizado.

Mesmo sendo uma xiloteca de pequeno porte, é de grande importância para a região, além de possuir representatividade, com 55 famílias depositadas, um número significativo de famílias de árvores (Figura 1) e diversos espécimes associados a estas. Segundo Stehmann et al. (2009), existem 13.708 espécies e 1.782 gêneros agrupados em 208 famílias de angiospermas conhecidas, não sendo nenhuma exclusiva da Floresta Atlântica; isso significa que quase $30 \%$ das famílias existentes estão presentes na coleção.

É fato que houve uma estabilização da xiloteca do CEPEC e que muitas xilotecas vêm sofrendo com fusão e/ou extinção de suas coleções (Barros; Coradin, 2016). Por isso, o objetivo atual é voltar a enriquecer, fortalecer, resgatar e aumentar a coleção de madeiras do CEPEC, incentivando novas coletas, não só na floresta Atlântica, mas, nas demais formações vegetacionais e regiões, estimulando trocas de conhecimento entre pesquisadores e estudantes.

Com o material biológico existente na coleção, é de interesse que estudos sejam realizados para a identificação das madeiras ainda não identificadas em nível de espécie, quando possível, e que sejam descritas suas propriedades organolépticas, físicas e anatômi- 
Tabela 1. Lista de espécimes da xiloteca do Centro de Pesquisas do Cacau - CEPEC -, Bahia, Brasil. Nomes científicos de acordo com Silva et al. (2008). N - número de exemplares; * - espécies exóticas e/ou cultivadas.

\begin{tabular}{|c|c|c|c|}
\hline Nome comum & Nome científico & $\mathbf{N}$ & Nome comum \\
\hline Abacate* & Persea americana Mill. & 1 & Claraíba-preta \\
\hline Abiu-roxo* & Chrysophyllum cainito $\mathrm{L}$. & 1 & Bacuparí \\
\hline Araçá-boi & Psidium rufum Mart.ex DC. & 1 & Bacuparí-preto \\
\hline Araçá-branco & Eugenia feijoi O.Berg. & 1 & Bálsamo \\
\hline Acá-de-leite & Pouteria sp. & 1 & Bálsamo-preto \\
\hline Aderno-faia-branco & Roupala sp. & 1 & Banha-de-galinha \\
\hline Aderno-faia-vermelho & Roupala brasiliensis Klotz. & 1 & $\begin{array}{l}\text { Banha-de-galinha } \\
\text { ou rapadura }\end{array}$ \\
\hline Aderno-vermelho & Emmotum nitens (Benth.) Miers & 1 & Bapeba \\
\hline Amendoeira* & Terminalia catapa $\mathrm{L}$. & 1 & Bapeba \\
\hline Amescla & Protium sp. & 1 & Bapeba \\
\hline Amescla-verdadeira & Protium heptaphyllum (Aubl.) Marchand & 3 & Bapeba-ferro \\
\hline Amora-preta & $\begin{array}{l}\text { Helicostyles tomentiosum (Poepp. \& Endl.) } \\
\text { Rusby }\end{array}$ & 4 & Bapeba-preta \\
\hline Angelim & Andira sp. & 1 & Bapeba-preta \\
\hline Angelim & Andira sp. & 1 & Bapeba-rosa \\
\hline Angelim & Andira sp. & 1 & Bapeba-vermelha \\
\hline Angelim-branco & Andira pisonis Mart. & 1 & Bassoré \\
\hline Angelim-coco & Andira anthelmia (Vell.) J. F. Macbr. & 1 & Bassoré-vermelho \\
\hline Araçá & Psidiuim sp. & 1 & Batinga \\
\hline Araçá-caboclo & Myrcia sp. & 1 & Batinguçú \\
\hline $\begin{array}{l}\text { Araçá/Araçá- } \\
\text { vermelho* }\end{array}$ & $\begin{array}{l}\text { Psidium guineense } \\
\text { Sw. Kuntze }\end{array}$ & 2 & Bicuíba-branca \\
\hline Araçá-d'água & $\begin{array}{l}\text { Terminalia brasiliensis (Cambess. ex A. St. } \\
\text {-Hil.) Eichl. }\end{array}$ & 1 & Bicuíba-vermelha \\
\hline Araribá-putumujú & $\begin{array}{l}\text { Centrolobium robustum (Vell.) Mart. ex } \\
\text { Benth. }\end{array}$ & 4 & Biriba-branca \\
\hline Arariba-branco & Simira sp. & 1 & Biriba-preta \\
\hline Arariba-cabacinha & $\begin{array}{l}\text { Macrothumia kublmannii (Sleumer) } \\
\text { M.H.Alford }\end{array}$ & 1 & Bomba-d'água \\
\hline Arariba-osso & sp. & 1 & Borboleta \\
\hline Arariba-rosa & Simira glaziiovii (K. Schum.) Steyerm. & 2 & Braúna \\
\hline Araroba & Vataireopsis araroba (Aguiar) Ducke & 2 & Buranhém \\
\hline Araroba ou Amargoso & Vataireopsis araroba (Aguiar) Ducke & 3 & Buranhém-branco \\
\hline Aroeira-de-remédio & Schinus terebinthifolia Raddi & 1 & Buranhém-pardo \\
\hline Baba-de-boi-branco & Cordia taguabyensisVell. & 1 & $\begin{array}{l}\text { Buranhém- } \\
\text { verdadeiro }\end{array}$ \\
\hline Baba-de-boi-preto & Cordia superba Cham. & 1 & Cabelo-de-cotia \\
\hline Bacumixá & Diploon cuspidatum (Hoehne) Cronquist & 1 & Cabelouro \\
\hline Bacumixá-branco & sp. & 1 & Cacau* \\
\hline Bacumixá-vermelha & sp. & 1 & Cajá* \\
\hline Carambola* & Avehoa sp. & 1 & Caju \\
\hline Carne-de-vaca & Roupala brasiliensis Klotz. & 1 & $\begin{array}{l}\text { Cajueiro-bravo/ } \\
\text { Cajueiro-bravo/ } \\
\text { Cajueiro-vermelho }\end{array}$ \\
\hline Carobinha & Jacaranda puberula Cham. & 1 & Camaçarí \\
\hline Carobinha & Jacaranda puberula Cham. & 1 & Cambucá \\
\hline Carrapato & Diospyros sp. & 1 & Esteieiro \\
\hline Carrapeta & $\begin{array}{l}\text { Macrothumia kublmannii (Sleumer) M.H. } \\
\text { Alford }\end{array}$ & 1 & Falso-Buranhém \\
\hline Casqueiro & Samanea sp. & 1 & Falso-Pau-Paraíba \\
\hline Cássia-negra & Cassia sp. & 1 & Farinha-seca \\
\hline $\begin{array}{l}\text { Cássia-rosa ou Cássia- } \\
\text { de-java* }\end{array}$ & Cassia javanica L. & 1 & Faveca-preta \\
\hline Cedro ou cedro-rosa & Cedrela odorata L. & 1 & $\begin{array}{l}\text { Faveca-vermelha ou } \\
\text { Arapati }\end{array}$ \\
\hline Cedro-arruda & sp. & 1 & Fidalgo \\
\hline Cedro-cabacinha & Guarea macrophylla Vahl & 1 & Flamboyant* \\
\hline Cedro-canjerana & Cabralea canjerana (Vell.) Mart. & 1 & Fruto-de-paca \\
\hline Cedro-vermelho & Cedrela macrocarpa Ducke & 1 & Gameleira-branca \\
\hline Cinzeiro & Rinorea guianensis Aubl. & 1 & Gameleira-preta \\
\hline Claraíba-parda & Cordia trichotoma (Vell.) Arrab. ex Steud. & 1 & Gindiba \\
\hline
\end{tabular}

\begin{tabular}{|c|c|}
\hline Nome científico & $\mathbf{N}$ \\
\hline Cordia sp. & 1 \\
\hline Garcinia macrophylla Mart. & 1 \\
\hline Garcinia sp. & 1 \\
\hline Myrocarpus fastigiatus Allemao & 1 \\
\hline Platymiscium sp. & 1 \\
\hline Guettarda platypoda DC. & 1 \\
\hline Guettarda sp. & 1 \\
\hline Pouteria spp. & 1 \\
\hline Pouteria spp. & 1 \\
\hline Pouteria spp. & 1 \\
\hline Pouteria spp. & 1 \\
\hline Chrysophyllum sp. & 1 \\
\hline Chrysophyllum sp. & 1 \\
\hline Pouteria spp. & 1 \\
\hline Chrysophyllum sp. & 1 \\
\hline Licania sp. & 1 \\
\hline Licania sp. & 1 \\
\hline Eugenia sp. & 1 \\
\hline sp. & 1 \\
\hline Virola officinalis (Mart.) Warb. & 3 \\
\hline Virola gardneri (A. DC.) Warb. & 1 \\
\hline Eschweilera ovata (Cambess.) Miers & 1 \\
\hline Eschweilera ovata (Cambess.) Miers & 1 \\
\hline Hydrogaster trinervis Kuhlm. & 2 \\
\hline Stephanopodium blanchetianum Baill. & 1 \\
\hline Melanoxylon brauna Schott & 1 \\
\hline Pradosiakublmannii Toledo & 2 \\
\hline sp. & 1 \\
\hline sp. & 1 \\
\hline sp. & 1 \\
\hline Miconia sp. & 1 \\
\hline Lonchocarpus neuroscapha Benth. & 1 \\
\hline Theobroma cacao L. & 1 \\
\hline Spondias mombin L. & 1 \\
\hline Anacardium occidentale L. & 1 \\
\hline Hyeromina alchorneoides Allemao & 3 \\
\hline Caraipa densifolia Mart. & 1 \\
\hline Marlierea edulis O. Berg & 2 \\
\hline Raputia paraenses Ducke & 2 \\
\hline sp. & 1 \\
\hline Simaba cedron Planch. & 1 \\
\hline Guapira spp. & 3 \\
\hline $\begin{array}{l}\text { Chamaecrista duartei H. S. Irwin) H. S. } \\
\text { Irwin \& Barneby }\end{array}$ & 1 \\
\hline $\begin{array}{l}\text { Arapatiella psilophylla (Harms) R. S. } \\
\text { Cowan }\end{array}$ & 2 \\
\hline Aegiphila integrifolia (Jacq.) Moldenke & 1 \\
\hline Delonix regia (Bojer ex Hook.) Raf. & 1 \\
\hline Carpotroche brasiliensis (Raddi) A. Gray & 1 \\
\hline Ficus clusiaefolia Scott & 1 \\
\hline Ficus castellvinna Dugand & 1 \\
\hline Sloanea obtusifolia (Moric.) K. Schum. & 4 \\
\hline
\end{tabular}


Tabela 1. Lista de espécimes da xiloteca do Centro de Pesquisas do Cacau - CEPEC -, Bahia, Brasil. Nomes científicos de acordo com Silva et al. (2008). N - número de exemplares; * - espécies exóticas e/ou cultivadas.

\begin{tabular}{|c|c|c|c|}
\hline Nome comum & Nome científico & $\mathbf{N}$ & Nome comum \\
\hline Cobí-preto & $\begin{array}{l}\text { Senna multijuga (L. C. Rich.) H. S. Irwin } \\
\text { \& Barneby }\end{array}$ & 1 & Gmelina* \\
\hline Cocão & $\begin{array}{l}\text { Pogonophora schomburgkiana Miers ex } \\
\text { Benth. }\end{array}$ & 2 & Goiabeira* \\
\hline Coco-da-Bahia & Cocos nucifera $\mathrm{L}$. & 1 & Gonçalo-vermelho \\
\hline Coco-danta & Amanoa guianensis Aubl. & 1 & Guabiraba-amarela \\
\hline $\begin{array}{l}\text { Conduru/Conduru- } \\
\text { vermelho }\end{array}$ & Brosimum rubescens Taub. & 2 & Guabiraba-verdadeira \\
\hline Cupuba & Tapirira guianensis Aubl. & 1 & Ingá \\
\hline Coração-de-negro & Swartzia apetala Raddi & 1 & Ingá-cipó \\
\hline Corindiba & Trema micrantha (L.) Blume & 1 & Ingá-da-mata \\
\hline Dandá ou Boleira & Joannesia princeps Vell. & 1 & $\begin{array}{l}\text { Ingauçú-amarelo/ } \\
\text { Ingauçú-branco/In- } \\
\text { gauçú-gigante }\end{array}$ \\
\hline Borboleta & Stephanopodium blanchetianum Baill. & 2 & Ingauçú-preto \\
\hline Embiruçú & $\begin{array}{l}\text { Eriotheca macrophylla } \\
\text { (K. Schum.) A. Robyns }\end{array}$ & 3 & Inhaíba \\
\hline Eritrina & Erythrina sp. & 1 & Inquirri-bravo \\
\hline Escada-de-macaco & Baubinia sp. & 1 & Ipê-amarelo \\
\hline Espatódea* & Spatodea sp. & 1 & Ipê-rosa \\
\hline Jacarandá-da-Bahia & $\begin{array}{l}\text { Dalbergia nigra } \\
\text { (Vell.) Allemao ex Benth }\end{array}$ & 1 & Jacarandá-branco \\
\hline Jacarandá-preto & $\begin{array}{l}\text { Machaerium floridum } \\
\text { (Mart. ex Benth.) Ducke }\end{array}$ & 1 & Jacarandá-da-Bahia \\
\hline Janaúba & $\begin{array}{l}\text { Himatanthus articulatus } \\
\text { (Vahl) Woodson }\end{array}$ & 1 & Jacarandá-preto \\
\hline Jangada-branca & Apeiba tibourbou Aubl. & 1 & Louro-prego \\
\hline Jaqueira* & $\begin{array}{l}\text { Artocarpus beterophyllus } \\
\text { Lam. }\end{array}$ & 1 & Louro-sassafrás \\
\hline Jatobá & Hymenaea courbaril L. & 1 & Maçaranduba-mirim \\
\hline Jatobá-peloso & Hymenaea sp. & 1 & Maçaranduba-praiú \\
\hline Jatobá-verdadeiro & $\begin{array}{l}\text { Hymenaea oblongifolia } \\
\text { Huber }\end{array}$ & 1 & $\begin{array}{l}\text { Maçaranduba- } \\
\text { verdadeira }\end{array}$ \\
\hline Jenipapo & Genipa americana L. & 1 & Mandioca-brava \\
\hline Jequitibá-cipó & $\begin{array}{l}\text { Cariniana estrellensis } \\
\text { (Raddi) Kuntze }\end{array}$ & 1 & Mangueira* \\
\hline Jequitibá-rosa & $\begin{array}{l}\text { Cariniana legalis } \\
\text { (Mart) Kuntze }\end{array}$ & 1 & Mangue-manso \\
\hline Jitaí-amarelo & $\begin{array}{l}\text { Apuleia leiocarpa } \\
\text { (Vogel) J.F.Macbr. }\end{array}$ & 1 & Mangue-vermelho \\
\hline $\begin{array}{l}\text { Jitaí-preto/ Jitaí- } \\
\text { vermelho }\end{array}$ & $\begin{array}{l}\text { Dialium guianense } \\
\text { (Aubl.) Sandwith }\end{array}$ & 2 & $\begin{array}{l}\text { Mangue-vermelho-da- } \\
\text { mata }\end{array}$ \\
\hline Juerana-branca & $\begin{array}{l}\text { Balizia pedicellare } \\
\text { (DC.) Barneby \& J. W. Grimes }\end{array}$ & 2 & Mata-cacau \\
\hline $\begin{array}{l}\text { Juerana-verdadeira ou } \\
\text { visgueira ou vermelha }\end{array}$ & $\begin{array}{l}\text { Parkia pendula } \\
\text { (Willd.) Benth. ex Walp. }\end{array}$ & 2 & Matataúba \\
\hline Kiri & sp. & 1 & Miroró-branco \\
\hline Laranjeira-brava & Zanthoxylum spp. & 1 & Miroró-de-embira \\
\hline Lava-pratos & $\begin{array}{l}\text { Alchornea glandulosa } \\
\text { Poepp. }\end{array}$ & 1 & Monzê \\
\hline Limão-balão* & Citrus aurantifolia L. & 1 & Mucitaíba \\
\hline Louro & Ocotea spp. & 4 & Mucitaíba-amarela \\
\hline Louro & Ocotea spp. & 1 & Mucitaíba-branca \\
\hline Louro-amarelo & Ocotea sp. & 2 & Mucitaíba-mongo \\
\hline Louro-cambraia & sp. & 1 & Mucitaíba-preta \\
\hline
\end{tabular}

\begin{tabular}{|c|c|}
\hline Nome científico & $\mathbf{N}$ \\
\hline Gmelina sp. & 1 \\
\hline Psidium guajava $\mathrm{L}$. & 1 \\
\hline sp. & 1 \\
\hline Campomanesia sp. & 1 \\
\hline Campomanesia sp. & 2 \\
\hline Inga spp. & 1 \\
\hline Inga vera Willd. & 1 \\
\hline Inga sp. & 1 \\
\hline Tachigali multijuga Benth. & 3 \\
\hline $\begin{array}{l}\text { Tachigali densiflorum } \\
\text { (Benth.) L.G.Silva \& H.C.Lima }\end{array}$ & 2 \\
\hline Lecythis lurida (Miers) Mori & 3 \\
\hline $\begin{array}{l}\text { Chrysophyllum ebenaceum } \\
\text { Mart. }\end{array}$ & 1 \\
\hline $\begin{array}{l}\text { Handroanthus chrysotrichus (Mart. ex } \\
\text { DC) Mattos }\end{array}$ & 1 \\
\hline $\begin{array}{l}\text { Handroanthus heptaphyllus (Vell.) Mat- } \\
\text { tos }\end{array}$ & 1 \\
\hline Swartzia macrostachya Benth. & 1 \\
\hline $\begin{array}{l}\text { Dalbergia nigra } \\
\text { (Vell.) Allemao ex Benth }\end{array}$ & 1 \\
\hline $\begin{array}{l}\text { Machaerium floridum } \\
\text { (Mart. ex Benth.) Ducke }\end{array}$ & 1 \\
\hline Nectandra sp. & 1 \\
\hline Ocotea pretiosa (Ness) Mez & 1 \\
\hline $\begin{array}{l}\text { Manilkara salzmanii } \\
\text { (A.DC.) H.J.Lam }\end{array}$ & 1 \\
\hline Manilkara maxima T.D.Penn. & 1 \\
\hline $\begin{array}{l}\text { Manilkara elata } \\
\text { (Allemao ex Miq.) Monach. }\end{array}$ & 2 \\
\hline $\begin{array}{l}\text { Manibot cf. pseudoglariovii Pax \& K. } \\
\text { Hoffm. }\end{array}$ & 1 \\
\hline Mangifera indica $\mathrm{L}$. & \\
\hline $\begin{array}{l}\text { Laguncularia racemosa } \\
\text { (L.) C. F. Gaertn. }\end{array}$ & 2 \\
\hline Rhirophora mangle $\mathrm{L}$. & 1 \\
\hline Tovomita mangle G. Mariz & 1 \\
\hline $\begin{array}{l}\text { Acanthosyris paulo-alvinii } \\
\text { G. M. Barroso }\end{array}$ & 1 \\
\hline $\begin{array}{l}\text { Schefflera morototoni } \\
\text { (Aubl.) Maguire et al. }\end{array}$ & 1 \\
\hline Guazuma crinita Mart. & 1 \\
\hline $\begin{array}{l}\text { Luebea grandiflora } \\
\text { Mart. \& Zucc. }\end{array}$ & 1 \\
\hline $\begin{array}{l}\text { Albizia polycephalum } \\
\text { (Benth.) Kilip ex Record }\end{array}$ & 1 \\
\hline $\begin{array}{l}\text { Zollernia ilicifolia } \\
\text { (Brongn.) Vogel }\end{array}$ & 2 \\
\hline Machaerium pedicellatum Vogel & 2 \\
\hline $\begin{array}{l}\text { Poecilanthe ulei (Harms) Arroyo \& } \\
\text { Rudd }\end{array}$ & 1 \\
\hline Acacia glomerosa Benth. & 1 \\
\hline $\begin{array}{l}\text { Zollernia ilicifolia } \\
\text { (Brongn.) Vogel }\end{array}$ & 2 \\
\hline
\end{tabular}


Tabela 1. Lista de espécimes da xiloteca do Centro de Pesquisas do Cacau - CEPEC -, Bahia, Brasil. Nomes científicos de acordo com Silva et al. (2008). N - número de exemplares; * - espécies exóticas e/ou cultivadas.

\begin{tabular}{|c|c|c|c|c|}
\hline Nome comum & Nome científico & $\mathbf{N}$ & Nome comum & Nome científico \\
\hline Louro-canela & Nectandra sp. & 1 & Pau-sangue & Pterocarpus rhorii Vahl \\
\hline Louro-casca-preta & sp. & 2 & Mucitaíba-rosa & Zollernia sp. \\
\hline Louro-cravo & Cryptocarya mandioccana Meisn. in DC. & 1 & Mucugê & Couma rigida Müll. Arg. \\
\hline Louro-d'água & Vochysia riedeliana Stafleu. & 2 & Mucuri & $\begin{array}{l}\text { Pouteria procera } \\
\text { (Mart.) K. Hammer }\end{array}$ \\
\hline Louro-graveto & Nectandra sp. & 2 & Mulungu & Erythrina fusca Lour. \\
\hline $\begin{array}{l}\text { Louro-pardo ou claraí- } \\
\text { ba-parda }\end{array}$ & Cordia trichotoma (Vell.) Arrab. ex Steud. & 1 & Mundururú & Miconia amoena Triana \\
\hline Louro-pimenta & Mequilaurus sp. & 2 & Murici & Byrsonima alvimii $\mathrm{W}$. R. Anderson \\
\hline Murta-branca & sp. & 1 & Mucrici-da-mata & Byrsonima stipulacea A. Juss. \\
\hline Murta-ferro & sp. & 1 & Murta & Eugenia florida DC. \\
\hline Murta-vermelha & sp. & 1 & Sombreiro & Clitoria fairchildiana R.A. Howard \\
\hline Murtim-bravo & Humiria balsamifera (Aubl.) J. St.-Hil. & 1 & Sucupira & $\begin{array}{l}\text { Diplotropis incexis, Rizzini \& A. } \\
\text { Mattos }\end{array}$ \\
\hline Murta-vermelha & sp. & 1 & $\begin{array}{l}\text { Sucupira-mirim/ Sucu- } \\
\text { pira-parda }\end{array}$ & Bowdichia virgilioides Kunth \\
\hline Oiti & Licania sp. & 2 & Sucupira-preta & $\begin{array}{l}\text { Chamaecrista apoucouita (Aubl.) } \\
\text { H.S.Irwin \& Barneby }\end{array}$ \\
\hline Oiti-boi & Licania salzmannii (Hook.f.) Fritsch & 1 & Sucupirucú & sp. \\
\hline Oiti-mirim & Sacoglottis mattogrossensis Malme & 1 & Tararanga & Pourouma velutina Mart. ex Miq. \\
\hline Oiti-pardo & $\begin{array}{l}\text { Hirtella hebeclada } \\
\text { Moric. ex DC. }\end{array}$ & 1 & Tararanga-de-lixa & Pourouma guianensis Aubl. \\
\hline Oiti-rosa & sp. & 1 & Teca* & Tectona grandis L. f. \\
\hline $\begin{array}{l}\text { Oiti-verdadeiro ou oiti } \\
\text {-boi }\end{array}$ & sp. & 1 & Timbuíba & Enterolobium Mart. \\
\hline Olandi & Symphonia globulifera L. f. & 1 & Trapia & Crataeva tapia $\mathrm{L}$. \\
\hline Óleo-Comumbá & Macrolobium rigidum R.S.Cowan & 2 & Umbaúba & Cecropia glaziovii Snethl. \\
\hline Óleo-de-copaíba & Copaifera multijuga Hayne & 1 & Unha-de-gavião & Ampelocera glabra Kuhlmann \\
\hline $\begin{array}{l}\text { Óleo-de-copaíba ou } \\
\text { Pau-óleo }\end{array}$ & Copaifera lucens Dwyer & 1 & Unha-de-vaca & Baubinia anacrostachia $\mathrm{L}$. \\
\hline Orelha-de-onça & Machaerium sp. & 1 & Vinhático & Plathymenia reticulata Benth. \\
\hline Palma-mater & sp. & 1 & Virote-bandeira & Quararibea turbinata (Sw.) Poir. \\
\hline Pau-Brasil & $\begin{array}{l}\text { Paubrasilia echinata Gagnon, H.C.Lima } \\
\text { \& G.P.Lewis }\end{array}$ & 1 & $\begin{array}{l}\text { Virote-preto/ Virote- } \\
\text { verdadeiro }\end{array}$ & $\begin{array}{l}\text { Quararibea floribunda (A. St.-Hil.) K. } \\
\text { Schum. }\end{array}$ \\
\hline Pau-canela & sp. & 1 & $\begin{array}{l}\text { Visgueiro ou Juerana- } \\
\text { prego }\end{array}$ & $\begin{array}{l}\text { Parkia pendula (Willd.) Benth. ex. } \\
\text { Walp. }\end{array}$ \\
\hline Pau-d'alho & Gallesia scorododendrum Casar & 1 & Xineném & sp. \\
\hline Pau-d'arco-branco & Tabebuia elliptica (DC.) Sandwith & 1 & Xineném-branco & sp. \\
\hline Pau-d'arco-peroba & Tabebuia roseo-alba (Ridl.) Sandwith & 1 & Jacarandá-da-Bahia & $\begin{array}{l}\text { Dalbergia nirgra (Vell.) Allemão ex } \\
\text { Benth }\end{array}$ \\
\hline Pau-d'arco-rosa & Tabebuia heptaphylla (Vell.) Toledo & 1 & Pau-roxo ou Roxinho & Peltogyne angustiflora Ducke \\
\hline Pau-d'óleo & Copaifera lucens Dwyer & 1 & Pau-sangue-amarelo & sp. \\
\hline Pau-marfim & Senefeldera multiflora Mart. & 2 & Pau-vela & sp. \\
\hline Pau-paraíba & Simarouba amara Aubl. & 3 & Seringueira* & $\begin{array}{l}\text { Hevea brasiliensis (Willd. ex A.Juss.) } \\
\text { Müll.Arg. }\end{array}$ \\
\hline Pau-paraíba-amarelo & sp. & 1 & Sete-capotes & Machaerium angustifolium Vogel \\
\hline Pau-pombo & Tapirira guianensis Aubl. & 3 & - & $\begin{array}{l}\text { Dalbergia decipularis Rizzini \& } \\
\text { A.Mattos }\end{array}$ \\
\hline
\end{tabular}


cas, já que a grande maioria foi registrada inicialmente apenas por nomes populares e esses nomes diferenciam-se entre regiões.

Além de tudo isso, as xilotecas têm importância fundamental para tecnologia, conservação dos recursos naturais e manejo de espécies florestais de importância econômica (Fonseca et al., 2005), uma vez que, com as diversas contribuições que as coleções podem fornecer, ajudam a aumentar a conscientização e o reconhecimento dos seus benefícios científicos e sociais (Vásquez-Correa, 2017).

\section{Quantidade de espécimes por família}

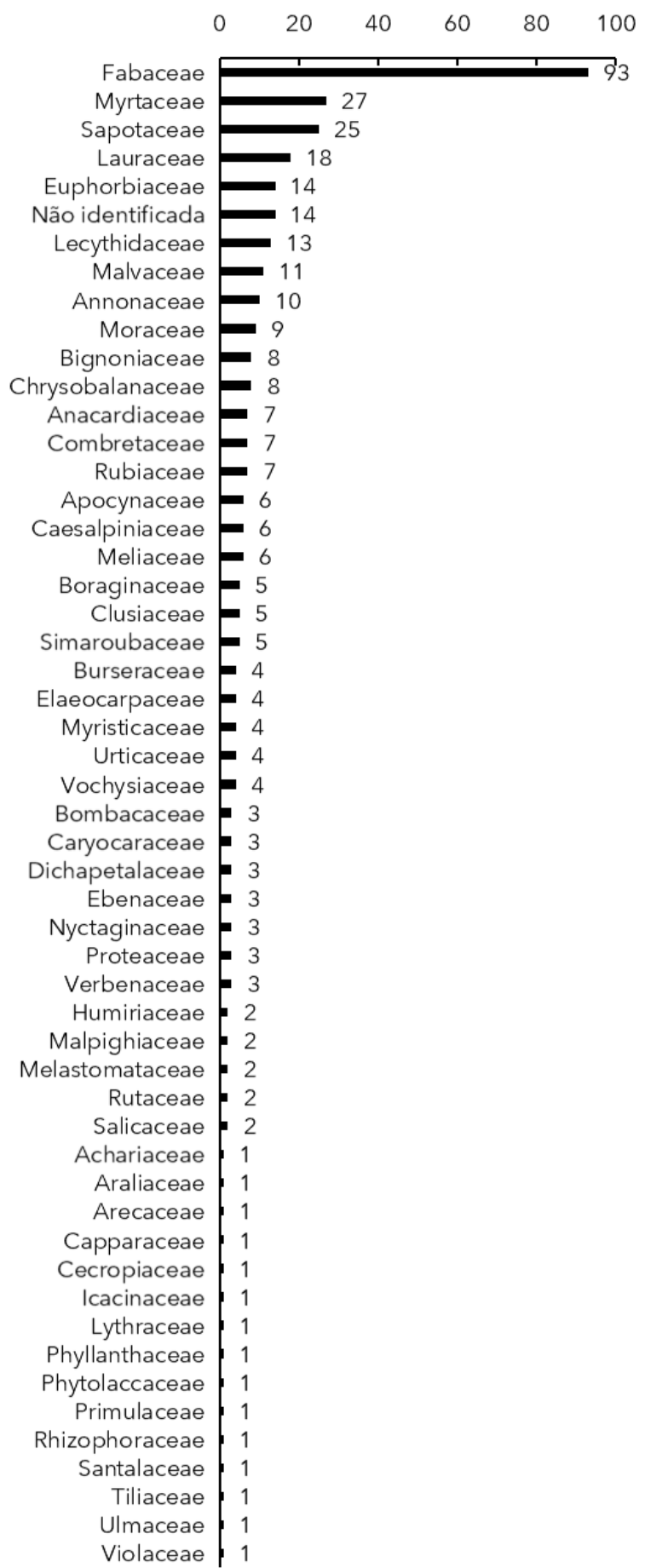

Figura 1. Famílias presentes na xiloteca do CEPECw e quantidade de espécimes por família.

\section{Conclusão}

O objetivo é que se faça um resgate da xiloteca e que sejam incorporadas mais amostras, com novas expedições, doações e permutas. O primeiro passo é a divulgação da xiloteca, dando visibilidade a pesquisadores do Brasil e estrangeiros. Dessa forma, temse a possibilidade de compartilhar informações antes restritas a um pequeno estrato de especialistas, popularizando assim o conhecimento. Uma próxima etapa será a confecção de um laminário para observação anatômica microscópica das madeiras, associado aos exemplares que ocorrem na xiloteca.

\section{Agradecimentos}

À Comissão Executiva do Plano da Lavoura Cacaueira - CEPLAC - e ao Centro de Pesquisa do Cacau - CEPEC - pelas facilitações em realizar o trabalho na xiloteca do CEPECw.

\section{Referências}

Amorim AM, Thomas WW, Carvalho AMV, Jardim JG. Floristic of the Una biological reserve, Bahia, Brazil. In: Thomas WW. The Atlantic coastal forests of North eastern Brazil. New York: Memoirs of the New York Botanical Garden; 2008. p. 67-146.

Amorim AM. Herbário Centro de Pesquisas do Cacau, Bahia, (CEPEC). Unisanta Bioscience 2015;4(6):98-101.

Barros CF, Coradin VTR. Xilotecas brasileiras: panorama atual e perspectivas futuras. Unisanta Bioscience 2016;4(7):29-40.

Dagnino R. Neutralidade da ciência e determinismo tecnológico. Campinas: Unicamp; 2008.

Fonseca CN, Lisboa PLB, Urbinati CV. A xiloteca (Coleção Walter A. Egler) do Museu Paraense Emílio Goeldi. Boletim do Museu Paraense Emílio Goeldi, série Ciências Naturais 2005;1(1):65-140.

Fozza RC, Baumgratz JF, Bicudo CE, Canhos D, Carvalho Junior AA, Coelho MN, Costa AF, Costa DP, Hopkins MG, Leitman PM, Lohmann LG, Luhandha EN, Maia LC, Martinelli G, Menezes M, Morim MP, Peixoto AL, Pirani JR, Prado J, QUEIROZ LP, SOUZA S, SOUZA VC, Stehmann JR, Sylvestres LS, Walter B, Zappi DC. New Brazilian floristic list highlights conservation challenges. BioScience 2012;62(1):39-45.

Lynch AH, Gasson PE. [Internet]. Index xylariorum 4.1 (updated by IAWA under supervision of Frederic Lens, Mar 2016). [acesso em 10 nov 2019]. Disponível em: https://www.iawawebsite.org/uploads/soft/Abstracts/Index\%20Xylariorum\% 204.1.pdf

Martini AMZ, Fiaschi P, Amorim AM, Paixão JP. A hot-point within a hot-spot: a high diversity site in Brazil's Atlantic forest. Biodiversity and Conservation 2007;16(11):3111-3128.

Mello Junior JCF, Amorim MW, Silveira ER. A xiloteca (coleção Joinvillea - JOIw) da Universidade da Região de Joinville. Rodriguésia 2014;65:1057-1060.

Mori AS, Silva LAM. O herbário do Centro de Pesquisas do Cacau em Itabuna, Brasil. Boletim Técnico 78. Comissão Executiva do Plano da Lavoura Cacaueira, 1979.

Mori SA, Boom BM, Prance GT. Distribution patterns and conservation of eastern Brazilian coastal forest tree species. Brittonia 1981;33(2):233-245.

Silva LAM, Jardim JG, Thomas WW, Santos TS. common names of vascular plants of the Atlantic coastal forest region of Southern Bahia, Brazil. In: Thomas WW. Atlantic coastal forest of North- 
eastern Brazil. New York: Memoirs of the New York Botanical Garden; 2008. p. 245-312.

Sobral M, Stehmann JR. An analysis of new angiosperm species discoveries in Brazil (1990-2006). Taxon 2009;58:227-232.

Stehmann JR, Forzza RZ, Salino A, Sobral M, Costa DP da, Kamino LHY (ed). Plantas da floresta Atlântica. Rio de Janeiro: Jardim Botânico do Rio de Janeiro; 2009.

Thomas WW, Carvalho AMV, Amorim AM, Garrison J, Arbeláez AL. Plant endemism in two forests in southern Bahia, Brazil. Biodiversity and Conservation 1998;7(3):311-322.

Vásquez-Correa AM. Xilotecas, importantes colecciones de referencia. Colombia Forestal 2017;20(2):192-201.

\section{La xiloteca del centro de investigación del ca- cao y las maderas del Bosque Atlántico}

Xiloteca es una colección cientifica de maderas identificadas y con datos de recolección disponibles, testigo de la diversidad biológica. Es una herramienta de referencia para estudios e investigaciones en el área botánica y tecnológica, tanto para productores como para comerciantes de madera, lo que permite la comparación e identificación de nuevas muestras de madera. La xiloteca descrita a continuación está asociada con el herbario del Centro de Investigación del Cacao, que actualmente es uno de los más grandes de la región noreste y posee la colección más importante de especimenes de plantas que representan el Bosque Atlántico del sur de Babia y el norte de Espirito Santo. El objetivo del estúdio es dar visibilidad a la información presente en la xiloteca. Con um aumento en la visibilidad de la xiloteca para um público más amplio investigadores y interessados en general -, esperamos mejorar la información que constituye el patrimonio de las especies vegetales en el sur de Babia, preservando asi datos de gran relevancia para la comprensión de la diversidad forestal de la región.

Palabras clave: Madera. Especies forestales. Colecciones botánicas. Colecciones de madera.

\section{La xylothèque du centre de recherche sur le cacao et les bois de la Forêt Atlantique}

Une xylothèque est une collection scientifique des bois identifiés avec des données de collecte disponibles, où la diversité biologique est représentée. $C^{\prime}$ est un outil de référence pour les études et les recherches dans les domaines botanique et technologique, à la fois pour les producteurs et les négociants en bois, et qui permet aussi la comparaison et l'identification de nouveaux échantillons de bois. La xylothèque en question est associée à l'berbier du Centre de recherches sur le Cacao, qui est actuellement l'un des plus importants du Nord-Est brésilien, et abrite la plus importante collection de spécimens de plantes représentatives de la forêt atlantique du sud de Babia et du nord de Espirito Santo. L'objectif de cette étude est de donner une plus grande visibilité aux informations présentes dans cette xylothèque. Avec l'augmentation de la visibilité de la xylothèque auprès d'un plus large public - des chercheurs et des personnes intéressées en général -, on prévoit valoriser les informations sur le patrimoine des espèces végétales du sud de Babia, en préservant ainsi des données d'une grande importance pour la comprébension de la diversité forestière de la région.

Mots clés: Bois. Espèces forestières. Collections botaniques. Collections de bois. 